\title{
Helicobacter pylori infection in children: relation with current household living conditions
}

\author{
W A McCallion, L J Murray, A G Bailie, A M Dalzell, D P J O'Reilly, K B Bamford
}

Departments of Paediatric Surgery A G Bailie

W A McCallion

and Paediatric

Medicine

A M Dalzell

The Royal Belfast Hospital for Sick Children, Belfast

Department of Epidemiology and Public Health L J Murray

and Microbiology and Immunobiology K B Bamford

The Queen's University of Belfast

Department of Public Health Medicine, Eastern Health and Social Services Board, Belfast

D P J O'Reilly

Correspondence to: Dr L Murray, Department of Social Medicine, Bristol University, Canynge Hall, Whiteladies Road, Bristol BS8 2PR

Accepted for publication 19 January 1996

\begin{abstract}
Background-Studies demonstrating that deprived household living conditions during childhood are risk factors for acquisition of Helicobacter pylori infection have been performed mainly in adults, who probably acquired the infection several decades ago. This study investigates whether deprived household living conditions remain important risk factors for infection in subjects (children) with recently acquired infection.
\end{abstract}

Aims-To examine the relation between current household living conditions and acquisition of $\boldsymbol{H}$ pylori infection in childhood.

Subjects/setting-Opportunistically recruited group of 367 children, aged 3 to 15 years, undergoing routine non-gastrointestinal day surgery.

Methods-Anti-H pylori IgG antibodies measured by a commercial enzyme linked immunosorbent assay validated for use in children. Postal questionnaire collecting sociodemographic data and data on household living conditions.

Results-Infection was associated with social class and overcrowding in the household. After adjustment for age, social class, and household density, a positive association remained between infection with $H$ pylori and bed-sharing between children and parents on one or two nights per week, odds ratio for infection $(95 \%$ CI, $2 \cdot 29(1 \cdot 21,4 \cdot 32)$ or more frequently, odds ratio for infection (95\% CI), $2 \cdot 95(1 \cdot 35,6 \cdot 45)$.

Conclusions-The continuing importance of household living conditions in the acquisition of $H$ pylori infection is confirmed and household crowding and sharing a bed with a parent are identified as risk factors for infection.

(Gut 1996; 39: 18-21)

Keywords: Helicobacter pylori, childhood, risk factors, breast feeding.

Helicobacter pylori is a spiral Gram negative bacterium that colonises the human stomach. It causes type B gastritis, ${ }^{1}$ is strongly associated with gastric and duodenal ulceration, ${ }^{2}$ and has been implicated in the causation of gastric carcinoma. ${ }^{34}$ No significant non-human or environmental reservoir of $H$ pylori infection has been identified indicating that person to person spread is almost certainly the mode of transmission of infection. Intra-familial clustering of infection is well recognised ${ }^{5}$ and the same strain of organism has been identified in parents and their offspring ${ }^{6}$ strongly suggesting the spread of infection occurs within the home. There is also a strong body of evidence showing that $H$ pylori infection is primarily acquired early in life. ${ }^{7-9}$ Further evidence for both acquisition of infection in childhood and spread within the home is provided by studies that have shown that deprived household living conditions in childhood such as overcrowding ${ }^{1011}$ and bed-sharing ${ }^{12}$ are risk factors for acquisition of $H$ pylori infection. A limitation of these studies, however, is that they have investigated adults who probably acquired the infection several decades ago: risk factors for infection acquired decades ago may be less important in recently acquired infection. It has been shown that, in developed countries, an age cohort effect exists ${ }^{13}$ and that the risk of acquisition of infection is decreasing with successive cohorts possibly as a result of improved living conditions in childhood. Recently investigators have suggested that, consequent upon improvements in household living conditions, factors in the community may now be more important in the transmission of $H$ pylori infection than conditions within the home. ${ }^{14}$ This study investigates whether deprived household living conditions remain important risk factors for infection in subjects (children) with recently acquired infection.

\section{Methods}

\section{Subjects}

Four hundred and eighty five children aged 3-15 years attending the Royal Belfast Hospital for Sick Children for routine nongastrointestinal day surgery were recruited over a six month period. Venepuncture was performed on the children under general anaesthesia. Serum (1 $\mathrm{ml})$ was stored at $-20^{\circ} \mathrm{C}$ and assayed in batches for anti- $H$ pylori IgG antibodies using a commercial enzyme linked immunosorbent assay (ELISA); Helico G, Porton Cambridge. The assay had previously been validated in 100 children undergoing routine upper gastrointestinal endoscopy, in whom three antral biopsy specimens had been taken for histology, culture, and rapid urease test (CLO-test, Delta West Ltd, Australia) respectively. A positive diagnosis of $H$ pylori infection was made if the organism was grown or there was both a positive CLO-test and histological evidence of gastritis. Using these criteria the sensitivity of the ELISA was $100 \%$ with a specificity of $92 \%$. 
TABLE I Unadjusted relations between variables and prevalence of seropositivity to $\mathrm{H}$ pylori

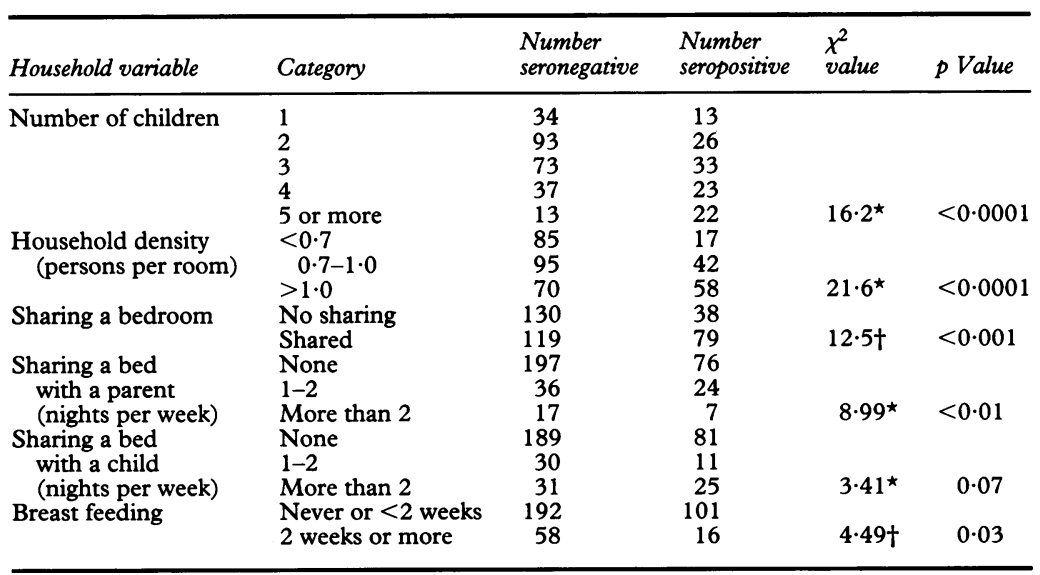

$\star=\chi^{2}$ for trend; $\dagger=\chi^{2}$. was used to examine the unadjusted relation between $H$ pylori infection and all variables. Multiple logistic regression (SPSS for Windows) was used to adjust for potential confounders.

This study was approved by the Research Ethics Committee of the Queen's University of Belfast. Informed parental consent was obtained in each case.

\section{Results}

Questionnaires were returned from 367 subjects, aged 3-15 years (response rate $76 \%$ ). One hundred and seventeen respondents were $H$ pylori seropositive (32\%); 42 non-respondents $(36 \%)$ were seropositive. Seroprevalence of $H$ pylori increased significantly with age; $21.4 \%$ of children aged less than 6 were seropositive, $29 \cdot 6 \%$ of those aged $6-8$ years old, $36.4 \%$ aged 9 to 11 years, and $52.8 \%$ aged 12 or over ( $\chi^{2}$ for trend $\left.16 \cdot 5, \mathrm{p}<0 \cdot 0001\right)$. Seropositivity was significantly more common in children whose parents were engaged in manual occupations $(38.3 \%$ infected) than in children whose parents were from non-manual occupations $\quad(23.5 \%$ infected $) ; \quad \chi^{2}=9.85$, $\mathrm{p}=0.002$.

Further bivariable analysis showed positive associations between $H$ pylori infection and the following household variables; number of children in the household, household density, sharing a bedroom, and sharing a bed with a parent/adult (Table I). There was a significant negative association between infection and breast feeding. Sharing a bed with a child was not significantly associated with infection but was significantly associated with household density $\left(\chi^{2}\right.$ for trend $\left.7 \cdot 0, \mathrm{p}<0.01\right)$. Sharing a bed with a parent was not associated with household density ( $\chi^{2}$ for trend $0.65, p=0.41$ ).

After adjusting for age and social class the following variables were significantly and positively associated with infection: number of children in the home (if five or more), household density, sharing a bedroom, sharing a bed with a child (on more than two nights a week), and sharing a bed with a parent (see Table II). On further adjustment for household density the only variable that remained significantly associated with infection was sharing a bed with a parent on one or two nights per week, odds ratio for infection (95\% CI), $2 \cdot 29(1 \cdot 21$, 4.32 ) or more frequently, odds ratio for infection $2.95(1.35,6.45)$. The odds ratio for infection in subjects sharing a bed with another child on more than two nights per week remained increased $(1.65(0.88,3.09)$ but the relation no longer achieved statistical significance. The odds ratio for infection in subjects who had been breast fed for two weeks or longer remained substantially decreased $(0.54(0.28,1.06))$ after adjustment for age, social class, and housing density but the relation did not achieve statistical significance $(0 \cdot 05<p<0 \cdot 1)$. Social class was strongly associated with infection on adjustment for age, odds ratio for infection in manual classes (95\% CI) $2.02(1.27,3.32)$, but after adjustment for household density and sharing a bed for less than two weeks and breast fed two weeks or longer. Household density was defined as the number of persons per room The distribution was positively skewed and was divided into three categories; less than $0 \cdot 7$ persons per room, 0.7-1 persons per room, and more than 1 person per room. The $\chi^{2}$ test (and where appropriate the $\chi^{2}$ test for trend) 
TABLE III The final logistic regression model, dependent variable $\mathrm{H}$ pylori infection

\begin{tabular}{|c|c|}
\hline Variable & Odds ratio \\
\hline $\begin{array}{l}\text { Age (y) } \\
\text { Social class (M: NM) } \\
\text { Household density (persons per room)^ }\end{array}$ & $\begin{array}{l}1.20(1 \cdot 10,1 \cdot 30) \\
1.60(0.97,2 \cdot 64)\end{array}$ \\
\hline $\begin{array}{l}0 \cdot 7-1 \\
>1\end{array}$ & $\begin{array}{l}2.02(1.04,3.92) \\
3.37(1.73,6.65)\end{array}$ \\
\hline $\begin{array}{l}\text { Sharing a bed with a parent/adult } \\
1 \text { or } 2 \text { nights per week } \\
\text { More than } 2 \text { nights per week }\end{array}$ & $\begin{array}{l}2 \cdot 29(1 \cdot 21,4 \cdot 32) \\
2.95(1 \cdot 35,6 \cdot 45)\end{array}$ \\
\hline
\end{tabular}

with a parent the relation between social class and infection was no longer statistically significant although the odds radio remained raised (Table III).

\section{Discussion}

Previous investigations in adults have shown that deprived household living conditions in childhood, particularly overcrowding ${ }^{10}$ and sharing a bed ${ }^{11} 12$ are associated with $H$ pylori infection. In this study we confirm the association between overcrowding in the household and infection with $H$ pylori in a group of children. High household density may present many opportunities for spread of infection through close personal contact. In many instances overcrowding will necessitate children sharing a bed and it is not clear whether sharing a bed is a risk factor for infection independently of overcrowding. Strong colinearity between overcrowding and sharing a bed resulted in Webb et al being unable to separate their effects. In this study although bed-sharing with another child and household density were closely related and each was associated with seropositivity for $H$ pylori infection when both variables were entered into the regression model household density remained strongly associated with infection while sharing a bed became nonsignificant (although the odds ratio for infection did remain increased). This suggests that sharing a bed with another child may be merely a measure of overcrowding in the household and not in itself a risk factor for infection.

In developed countries, $H$ pylori infection is more common in adults than in children and acquisition of infection primarily occurs in childhood suggesting that infection is transmitted from adult to child. Familial clustering of infection and isolation of the same strain of organism in adults and their children provide evidence that infection may be transmitted from infected parent to the child presumably within the household (although transmission of infection from infected child to uninfected adult is also a possibility). Close personal contact such as the sharing of a bed between a parent/adult and a child may provide the opportunity for transmission of infection from parent to child. Studies to date have not differentiated between sharing a bed during childhood with another child and sharing a bed with a parent/adult. In this study parents or guardians were asked if the child spent part of the night in their bed on one or more nights during the week. One in four children shared a bed with a parent/adult and this was not related to household overcrowding or social class. Within this group, unlike sharing a bed with another child, sharing a bed with a parent was a strong and independent risk factor for infection. Thus the mechanism of spread of infection from parent to child may be through close personal contact, for example, while sharing a bed.

It is interesting, but perhaps not surprising, that the relation between social class and infection becomes non-significant after adjustment for household density and bed-sharing between a child and an adult, suggesting that, with regard to acquisition of $H$ pylori infection, social class is acting as a proxy measure for conditions and practices within the household that increase the likelihood of transmission of the organism from infected to uninfected subject. It is also probable that there are other unidentified factors that contribute to the social class gradient in infection as the magnitude of the odds ratio remained increased after adjustment for household crowding and bed-sharing.

Anti- $H$ pylori IgA in human breast milk has been shown to protect against early acquisition of $H$ pylori infection in infants in a developing country ${ }^{16}$ and in this study breast fed children had a lower prevalence of infection than those bottle fed. This relation did not remain statistically significant after adjustment for confounders; age, social class, and household density. However, as fewer than $25 \%$ of the children were breast fed for two weeks or longer and as the magnitude of the effect and the direction of the relation were substantially unaltered with adjustment, one cannot rule out a true protective effect of breast feeding.

The opportunistic group of children studied was not population representative and findings within the group cannot be considered to be directly applicable to children who do not attend hospital for elective day surgery. However, as children from all social classes and from all parts of Northern Ireland were studied, the risk factors for acquisition of infection seen within this group may accurately reflect risk factors in the general population.

In conclusion this study confirms the continuing importance of household living conditions in the acquisition of $H$ pylori infection and identifies household crowding and sharing a bed with a parent during childhood as risk factors for infection. There is also some evidence that breast feeding may reduce the risk of infection.

We thank the following staff of the Royal Belfast Hospital for Sick Children: Mr V E Boston, Mr S R Potts, and Mr S Brown, consultant paediatric surgeons; $\mathrm{Mr} \mathrm{T}$ Gregg and $\mathrm{Mr}$ I consultant paediatric surgeons; $\mathrm{Mr}$ T Gregg and $\mathrm{Mr}$

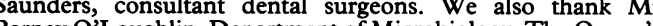

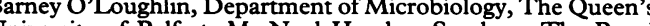
University of Belfast; Mr Noel Heasley, Serology, The Roya Victoria Hospital, Belfast and Dr Ian Harvey, Department of Social Medicine, The University of Bristol.

1 Warren JR. Unidentified curved bacteria on gastric epithelium in active chronic gastritis. Lancet 1983; $i$ : 1311-4.

2 DeCross AJ, Marshall BJ. The role of Helicobacter pylori in acid-peptic disease. Am $\mathcal{F}$ Med Sci 1993; 306: 382-92.

3 Parsonnet J, Friedman GD, Vandersteen DP, Chang Y Parsonnet J, Friedman GD, Vandersteen DP, Chang Y,
Vogelman JH, Orentreich H, et al. Helicobacter pylori infection and the risk of gastric carcinoma. $N$ Engl $\Im$ Med 1991; 325: 1127-31. 
4 The Eurogast Study Group. An international association between Helicobacter pylori infection and gastric cancer. Lancet 1993; 341: 1359-62.

5 Drumm B, Perez-Perez GI, Blaser MJ, Sherman PM Intrafamilial clustering of Helicobacter pylori infection. $N$ Intrafamilial clustering of Helicobacter pylori infection. $N$ Engl f Med 1990; 322: 359-63.

Bamford KB, Bickley J, Collins JSA, Johnston BT, Potts S Boston V, et al. Helicobacter pylori: comparison of DNA fingerprints provides evidence for intrafamilial infection. Gut 1993; 34: 1348-50.

7 Parsonnet J, Blaser MJ, Perez-Perez GI, Hargrett-Bean N, Tauxe RV. Symptoms and risk factors of Helicobacter pylori infection in a cohort of epidemiologists. Gyastroenterology 1992; 102: 41-6.

8 Cullen DJE, Collins BJ, Christiansen KJ, Epis J, Warren JR, Surveyor I, Cullen KJ. When is Helicobacter pylori infection acquired? Gut 1993; 34: 1681-2.

9 McCallion WA, Ardill JES, Bamford KB, Potts SR, Boston VE. Age dependent hypergastrinaemia in children with Helicobacter pylori gastritis - evidence of early acquisition Helicobacter pylori gastritis - eviden
of infection. Gut 1995; 37: 35-8

10 Mendall MA, Goggin PM, Molineaux N, Levy J, Toosy T Strachan D, et al. Childhood living conditions and
Helicobacter pylori seropositivity in adult life. Lancet 1992; 339: 896-7.

11 Whitaker CJ, Dubiel AJ, Galpin OP. Social and geographical risk factors in Helicobacter pylori infection. Epidemiol Infect 1993; 111: 63-70.

12 Webb PM, Knight T, Greaves S, Wilson A, Newell DG Elder J, et al. Relation between infection with Helicobacter pylori and living conditions in childhood: evidence for person to person transmission in early life. BMF 1994; 308: 750-3.

13 Banatvala N, Mayo K, Megraud F, Jennings R, Deeks JJ Feldman RA. The cohort effect and Helicobacter pylori. $f$ Infect Dis 1993; 168: 219-21.

14 Patel P, Mendall MA, Khulusi S, Northfield TC, Strachan DP. Helicobacter pylori infection in childhood; risk factors and effect on growth. BMF 1994; 309: 1119-23.

15 Office of Population Censuses and Surveys. Classification of occupations. London: Government Statistical Service, 1980.

16 Thomas JE, Austin S, Dale A, McClean P, Harding M, Coward WA, et al. Protection by human milk IgA against Helicobacter pylori infection in infancy [Letter]. Lancet 1993; 342: 121 . 